\title{
Proposed changes for substance use and gambling disorders in DSM-5: impact on assessment and treatment in the UK
}

\author{
Nancy M. Petry, ${ }^{1}$ Henrietta Bowden-Jones, ${ }^{2}$ Sanju George ${ }^{3}$
}

The Psychiatrist (2013), 37, 41-43, doi: 10.1192/pb.bp.112.040063

${ }^{1}$ University of Connecticut School of Medicine, USA; ${ }^{2}$ National Problem Gambling Clinic and Imperial College London, UK; ${ }^{3}$ Solihull Integrated Addiction Services and Birmingham \& Solihull Mental Health NHS Foundation Trust, UK

Correspondence to Nancy M. Petry (npetry@uchc.edu)

First received 10 May 2012, final

revision 18 Jul 2012, accepted 2 Aug 2012
Summary In the USA, DSM-5 is scheduled for publication in 2013. It is likely to include some substantial changes to the diagnosis of substance use disorders and pathological gambling. This paper reviews the proposed changes to these diagnoses and their potential impact on diagnosis, treatment and research of these disorders in the UK.

Declaration of interest N.M.P. is a member of the DSM-5 Substance-Related Disorders Work Group.
The DSM is the primary classification system for diagnosing psychiatric disorders in the USA. It is widely used in other countries as well, including the UK, although the other major classification system, ICD-10, is also used. The fifth edition of the DSM (DSM-5), scheduled for publication in 2013 , is likely to include changes to the diagnosis of substance use disorders, some of which are more aligned with ICD-10 criteria. In addition, DSM-5 will include changes to a particular related disorder - pathological gambling. This article summarises proposed changes to this section of the DSM, and it describes their potential impact on diagnosis and treatment of substance use and gambling disorders in the UK.

\section{Substantive changes to diagnosis of substance use disorders}

The most substantial change recommended for substance use disorders is to eliminate the 'abuse' versus 'dependence' dichotomy. In DSM-IV, abuse is diagnosed when an individual endorses one or more of four criteria related to adverse social and interpersonal consequence of heavy substance use. A diagnosis of dependence is rendered for meeting three or more of seven criteria, which assess physiological and other consequences of heavy alcohol or drug use. The division between abuse and dependence was premised on the concept that abuse was a milder form of dependence. However, analyses from over 30 different studies, consisting of over 200000 persons, revealed that 10 of the 11 DSM-IV substance use criteria form a unidimensional structure (e.g. Hasin et al, ${ }^{1}$ Saha et $a l^{2}$ ). The four abuse criteria are not associated with less severe forms of the disorder, and instead span across the severity spectrum.
The only criterion that did not fit with the others and added little diagnostic information was that related to committing illegal acts. ${ }^{1-3}$ This criterion has low prevalence rates, varies depending on the cultural context, and it is rarely endorsed in the absence of other criteria. Thus, a recommendation is to remove the illegal acts criterion in DSM-5.

One criterion is being recommended for inclusion. Strong desires to use or craving is a dependence criterion in ICD-10. The DSM-5 Substance-Related Disorders Work Group evaluated a number of cross-cultural epidemiological and clinical data-sets in which craving was assessed along with the other substance use disorder criteria. Craving fit with the other criteria and did not impact severity, discrimination or factor loadings. ${ }^{1,3}$ For consistency with ICD-10 and because of support for inclusion of this criterion among many clinicians, craving may be included in the diagnosis of substance use disorders in DSM-5.

Thus, 11 criteria are proposed for substance use diagnoses in DSM-5, requiring consideration of the optimal threshold for diagnosis. Although abuse was diagnosed by meeting just one of four criteria in DSM-IV, a single criterion is not a syndrome. The DSM-5 Substance-Related Disorders Work Group evaluated data from epidemiological and clinical samples to determine agreement with DSM-IV diagnoses across thresholds ranging from $\geqslant 2$ to $\geqslant 4$ criteria. Because agreement was very good to excellent using a threshold of $\geqslant 2$ criteria, it was selected. Although some have argued that a threshold of $\geqslant 2$ criteria is too inclusive, this concern needs to be balanced with the need to diagnose - and treat - milder cases such as patients presenting in primary care settings who have underlying substance misuse problems. Severity indicators are proposed, such that meeting only two criteria represents a milder form of the disorder. 


\section{Substantive changes proposed for pathological gambling}

In addition to changes in the diagnosis of substance use disorders, the chapter is likely to be broadened to include behavioural (or non-substance) addictions, such as pathological gambling. In DSM-IV and earlier editions, pathological gambling was classified in the Impulse-Control Disorders Not Elsewhere Classified section. Consistently high rates of comorbidity between substance use disorders and pathological gambling, genetic and physiological overlap, and similarities in presentation of some symptoms and treatment approaches led to the decision to include gambling alongside substance use disorders in DSM-5. ${ }^{4-8}$ With this move, the name of the disorder will also be altered to 'gambling disorder'. Other terms such as 'compulsive gambling' and 'problem gambling' were considered. However, the term 'compulsive' is commonly applied to disorders associated with anxiety, and 'problem gambling' has often been used to refer to a subdiagnostic threshold condition and therefore may unintentionally minimise the severity of the disorder.

Similarly to substance use disorders, the diagnostic criteria for gambling were examined with respect to their fit and classification accuracy in epidemiological and clinical samples. In DSM-IV, five of ten criteria are needed for diagnosis. The least prevalent criterion is the one related to committing illegal acts to gamble, such as forgery, fraud, theft or embezzlement, and this criterion is rarely endorsed in the absence of other criteria. ${ }^{9}$ Further, statistical analyses revealed that this item adds little to classification accuracy and it is therefore being recommended for deletion in DSM-5, similarly to the parallel criterion for substance use disorders.

The threshold for diagnosis was also considered. Independent analyses revealed that reducing the threshold to four criteria improved classification accuracy (e.g. Stinchfield ${ }^{10}$ ). Hence, the recommendation is that a gambling disorder diagnosis will constitute meeting four of nine criteria in DSM-5, and this change is unlikely to impact base rates substantially. ${ }^{11}$

\section{Other non-substance use addictive disorders}

Several other putative conditions were considered for inclusion in this section. A subcommittee convened to assess the literature related to internet/gaming addiction, compulsive shopping, and several other behaviour patterns. The Substance-Related Disorders Work Group concluded that sufficient data do not yet exist to classify any of these conditions as mental disorders in DSM-5. Although a large literature exists on gaming addiction, no consensus exists regarding how the condition is best classified. Further, limited data are available regarding the prevalence or clinical course of the putative disorder. As more research is conducted, empirical data will guide decisions about whether and how best to diagnose such conditions for future editions of DSM, and gaming addiction is likely to be listed in the appendix as a potential disorder requiring more research.

\section{How these changes may impact diagnosis and treatment in the UK}

Despite the wide ranging use of DSM throughout the world, medical students in the UK are trained using ICD-10, and doctors across the country classify most psychiatric disorders using this manual. One exception relates to gambling. Although few physicians or researchers in the UK diagnose or treat gambling, physicians in practice as well as in the National Problem Gambling Clinic have officially adopted the DSM criteria for this disorder. Using the DSM criteria has allowed for greater collaboration in international research projects.

The changes in diagnoses with DSM-5 are unlikely to impact the classification of substance use disorders clinically or with respect to research in the UK, given the reliance on ICD-10 for these purposes. However, the changes proposed for gambling disorder, especially with regard to the placement of gambling disorder in a similar section with other addictive disorders, are generally welcome. The inclusion of gambling disorder in this section is likely to lend greater credibility to the concept of excessive gambling as a psychiatric disorder. Clinically, this change may encourage more screening efforts for gambling in primary care and substance misuse treatment clinics, which in turn may lead to greater recognition of the need for brief interventions for disordered gambling in high-risk patient populations. ${ }^{8}$ Greater awareness and recognition of gambling disorder can assist both clinicians and researchers to communicate better with policy makers, commissioners of services and research funders of the need to invest more in this field and expand treatment services to those with gambling disorder. Further, if the ICD follows the DSM lead on classifying gambling disorder alongside substance use disorders, physicians in training and clinicians in practice may be more likely to place it at par with substance use disorders. This change is long overdue.

\section{Funding}

Preparation of this report is based in part on National Institutes of Health grants R01-DA021567, R01-MH60417, P30-DA023918, R01-DA027615, R01-DA022739, R01-DA13444, R01-DA018883, R01-DA016855, R01DA14618, P50-DA09241, P60-AA03510, and R01-DA024667.

\section{About the authors}

Nancy M. Petry, Professor of Medicine, University of Connecticut School of Medicine, USA. Henrietta Bowden-Jones, Director and Lead Clinician, National Problem Gambling Clinic and Imperial College, UK. Sanju George, Consultant in Addiction Psychiatry, Solihull Integrated Addiction Services and Birmingham \& Solihull Mental Health NHS Foundation Trust, UK

\section{References}

1 Hasin DS, Fenton MC, Beseler C, Park JY, Wall MM. Analyses related to the development of DSM-5 criteria for substance use related disorders: proposed DSM-5 criteria for alcohol, cannabis, cocaine and heroin disorders in 663 substance abuse patients. Drug Alcohol Depend 2012; 122: 28-37.

2 Saha TD, Compton WM, Chou SP, Smith S, Ruan WJ, Huang B, et al. Analyses related to the development of DSM-5 criteria for substance use related disorders: toward amphetamine, cocaine and prescription 
drug use disorder continua using Item Response Theory. Drug Alcohol Depend 2012; 122: 38-46.

3 Agrawal A, Heath AC, Lynskey MT. DSM-IV to DSM-5: the impact of proposed revisions on diagnosis of alcohol use disorders. Addiction 2011; 106: $1935-43$

4 Hodgins DC, Stea JN, Grant JE. Gambling disorders. Lancet 2011; 378: 1874-84.

5 Petry NM. Should the scope of addictive behaviors be broadened to include pathological gambling? Addiction 2006; 101: 152-60.

6 Petry NM, Ammerman Y, Bohl J, Doersch A, Gay H, Kadden R, et al. Cognitive-behavioral therapy for pathological gamblers. J Consult Clin Psychology 2006; 74: 555-67.

7 Petry NM, Stinson FS, Grant BF. Comorbidity of DSM-IV pathological gambling and psychiatric disorders: results from the National
Epidemiologic Survey on Alcohol and Related Conditions. J Clin Psychiatry 2005; 66: 564-74.

8 Petry NM, Weinstock J, Morasco BJ, Ledgerwood DM. Brief motivational interventions for college student problem gamblers. Addiction 2009; 104: 1569-78.

9 Strong DR, Kahler CW. Evaluation of the continuum of gambling problems using the DSM-IV. Addiction 2007; 102: 713-21.

10 Stinchfield R. Reliability, validity, and classification accuracy of a measure of DSM-IV diagnostic criteria for pathological gambling. Am J Psychiatry 2003; 160: 180-2.

11 Denis C, Fatséas M, Auriacombe M. Analyses related to the development of DSM-5 criteria for substance use related disorders: an assessment of Pathological Gambling criteria. Drug Alcohol Depend 2012; 122: 22-7. 